\title{
El proceso de convergencia de las enseñanzas de arte dramático y danza en el Espacio Europeo de Educación Superior
}

\author{
María Matilde Pérez García ${ }^{1 *}$ y Álvaro Sicilia Camacho \\ ${ }^{1}$ Conservatorio Superior de Danza de Málaga \\ ${ }^{2}$ Universidad de Almería
}

(Recibido 15 Septiembre 2011, Aceptado 21 Octubre 2011)

\begin{abstract}
RESUMEN: Ante la reforma de la educación superior en España para conseguir la convergencia al Espacio Europeo de Educación Superior (EEES), las enseñanzas artísticas se sitúan ante un novedoso y doble desafío. Por un lado, se precisa de la estructuración y diseño de un marco de ordenación y funcionamiento que permita el desarrollo de las enseñanzas artísticas en enseñanzas superiores. De otro, se requiere la elaboración y puesta en marcha de planes de estudio que han de ser evaluados y acreditados por las agencias de aseguramiento de la calidad. A partir del análisis de este doble desafío, el artículo sugiere algunas propuestas que la administración, los centros de enseñanzas artísticas y el profesorado podrían tener en cuenta de cara a la especial situación que estos estudios están viviendo en el proceso de convergencia europea.
\end{abstract}

Palabras clave: cambio educativo, educación superior, educación artística.

The convergence process of dramatic art and dance studies in the European Higher Education Area

\begin{abstract}
In light of the reform of Spanish higher education in order to achieve higher education European convergence, art studies face a new and twofold challenge. On the one hand, the design and structure of an organizational and operational framework is necessary to allow for the development of art studies in higher education. On the other hand, the design and implementation of curricula is required which have to be evaluated and accredited by agencies to ensure sufficient quality of education. From the analysis of this twofold challenge, the paper suggests some proposals that the administration, the schools and the staff could consider with regard to the special situation that these studies are going through in the process of European convergence.
\end{abstract}

Keywords: educational change, higher education, art education.

\section{INTRODUCCIÓN}

En la actualidad, no solo España, sino la mayoría de los países y sus sistemas de educación superior se están preparando o han experimentado cambios evidentes, siendo el grado en el que operan muy diferente (Lemaitre, 2004). El último intento que nos afecta ha sido la Declaración de Bolonia de 1999, poniendo en marcha el Espacio Europeo de Educación Superior (EEES). 
Cualquier momento de cambio puede traer asociadas numerosas incertidumbres, pero también la posibilidad de cambiar determinadas cuestiones que no gustan, que no funcionan, o que no son adecuadas. Si para la propia universidad española la convergencia con el EEES está aportando un debate profundo sobre estos procesos y sus consecuencias, en el caso de las enseñanzas artísticas este debate está sirviendo para la implantación definitiva de estos estudios en la educación superior.

Los centros de enseñanza superior deberán ser capaces de elaborar planes de estudio de grado y postgrado que cubran las necesidades de la sociedad, otorgando y reconociendo cualificaciones con valor en el mercado laboral que dan derecho a ejercer una profesión (Vélaz de Medrano, 2008). En esta reforma de la educación superior en España, las enseñanzas artísticas se sitúan ante un doble desafío. Por un lado, las enseñanzas artísticas necesitan un nuevo marco estructural que les permita el desarrollo de sus enseñanzas como enseñanzas superiores, situación que hasta el momento se ha visto limitada. Por otro lado, las enseñanzas artísticas necesitan elaborar y poner en marcha planes de estudios acordes con el nuevo marco estructural de la enseñanza superior y la convergencia europea, que deberán ser evaluados y acreditados por la agencias de calidad.

El presente artículo revisa el momento crucial que las enseñanzas de arte dramático y enseñanzas superiores de danza viven en España ante este doble reto. A partir de este análisis, se sugieren algunas actuaciones que podrían facilitar el proceso de convergencia europea de la educación superior en las enseñanzas artísticas. Así, el artículo se estructura en tres partes. En las primeras dos secciones se aborda la situación estructural y curricular de las enseñanzas superiores de arte dramático y danza, para finalizar sugiriendo algunas actuaciones que desde las esfera administrativa, los centros educativos y los profesores e investigadores pueden llevarse a cabo.

\section{EL DESAFÍO ESTRUCTURAL DE LAS ENSEÑANZAS ARTÍSTICAS SUPERIORES: DE LA LOGSE AL ESPACIO EUROPEO DE EDUCACIÓN SUPERIOR}

Las enseñanzas artísticas superiores (EEAASS) en España se enfrentan al proceso de convergencia europea en condiciones bastantes más limitadas al que lo hace la propia universidad. Debemos tener en cuenta que las enseñanzas artísticas en España se han desarrollado tradicionalmente en un territorio marginal dentro de los sistemas generales de educación. Por regla general, las enseñanzas artísticas en nuestro país han sido sostenidas por sistemas gremiales dependientes de la iglesia o de la nobleza y, por tanto, fuera de la tutela y la supervisión institucional necesaria (Zaldívar, 2005).

Las enseñanzas de danza y arte dramático en España, aunque con trayectorias legislativas muy distintas, se igualaron al regularse como enseñanzas de grado superior (equivalencia a licenciatura universitaria), en la Ley Orgánica de Ordenación General del Sistema Educativo 1/1990, de 3 de octubre (LOGSE). La 
LOGSE fue el resultado de varios años de experimentación y de debate sobre los objetivos y las características que debía presentar el sistema educativo español en las siguientes décadas (Marchesi, 2001; Pastora, 2000). Fue un intento de modernización y de adaptación de determinadas directrices europeas en materia de educación, y se configuró como las bases de todas las reformas educativas posteriores (Beltrán, Hernández y Montané, 2008). La ubicación de las enseñanzas de danza y arte dramático en este marco quedaron dentro de las enseñanzas artísticas, que a su vez se ubicaron dentro de las enseñanzas de régimen especial, junto a las enseñanzas superiores de música.

Esta nueva concepción educativa supuso un avance para el reconocimiento de las EEAASS en nuestro país, y dignificó las profesiones artísticas a través de un reconocimiento oficial de sus estudios dentro, y no al margen, del sistema educativo español. Sin embargo, al tratarse de unas enseñanzas minoritarias, en algunas comunidades autónomas, se ha retrasado el ingreso del profesorado al funcionariado y a un estatus profesional común. Por ejemplo, en el año 2008, sólo el 14\% aproximadamente del profesorado en las Escuelas de Arte Dramático de Andalucía era funcionario. En el mismo sentido, Jordi Font (2010), apunta la desuniformidad de estatus y las pretensiones frustradas del profesorado de las EEAASS ante las inalcanzables cátedras universitarias.

A pesar del novedoso impulso que la LOGSE otorgó a las enseñanzas artísticas, el marco general de actuación dependió de las autonomías, lo que ha contribuido a una falta de uniformidad y menor autonomía en su desarrollo respecto al que ya presentaban otras enseñanzas superiores. De hecho, en la situación actual puede afirmarse que las EEAASS se imparten en la mayoría de las comunidades desde unas estructuras similares a las de la enseñanza secundaria. De este modo, se muestran importantes incongruencias y contradicciones en la organización y funcionamiento de los centros, la labor investigadora, los intentos de movilidad internacional del profesorado y alumnado, etc. Algunos autores han considerado que esta desatención a las enseñanzas artísticas durante un gran periodo de tiempo, tanto desde el gobierno como desde la mayor parte de las autonomías, ha sido consecuencia de una falta generalizada de interés político, ya que éstas representan una pequeña, heterogénea e incómoda parte del sistema educativo (Alejandre, 2010; Pliego de Andrés, 2008). Lo que parece lógico, tal y como sugiere Zaldívar (2005), es que el modelo organizativo de las EEAASS debería ser homologable al universitario, pues conducen a la misma titulación.

La falta de adaptación de la estructura y funcionamiento, en el camino de convertirse en enseñanzas superiores y cumplir los criterios de la convergencia europea, ha estado influida también por la falta de acuerdo político sobre su futuro. De hecho, la incertidumbre de las enseñanzas artísticas se hacía evidente con la promulgación, en 2002, de la Ley Orgánica 10/2002 de Calidad de la Educación (LOCE), donde se obviaban referencias a la ordenación superior de las enseñanzas artísticas; quedando de nuevo estas enseñanzas relegadas a un estatus similar al de la educación secundaria. Sin embargo, la LOCE fue sustituida por la Ley Orgánica 2/2006, de Educación (LOE), con una visión diferente respecto 
a las enseñanzas artísticas. Esta ley volvió a situarlas, al menos sobre el papel, en el sistema de educación superior.

Desde la implantación de la LOE han existido diferentes Reales Decretos que la han desarrollado, con cuestiones de interés para las EEAASS y su integración en el Espacio Europeo. El Real Decreto 365/2007, de 16 de marzo, regula el Consejo Superior de las Enseñanzas Artísticas, como órgano de consulta y participación. En él se establece la plena integración de las EEAASS en el EEES, donde entre otras, se encuentran las enseñanzas superiores de danza y de arte dramático. Igualmente, el Real Decreto 1614/2009, de 26 de octubre, establece la ordenación de las EEAASS reguladas por la LOE. También regula y estructura las enseñanzas de acuerdo con el marco europeo de grados y posgrados. Asimismo, este Real Decreto recoge el fomento del Programa de Movilidad Europeo Erasmus para estudiantes y profesores, los créditos ECTS y la expedición del suplemento europeo al título.

El desarrollo de la LOE se ha completado recientemente con nuevos Reales Decretos. Entre ellos destacamos, por un lado, el Real Decreto 303/2010, de 15 de marzo, por el que se establece los requisitos mínimos de los centros que imparten enseñanzas artísticas y, por otro, los Reales Decretos 630/2010 y 632/2010, de 14 de mayo, que regulan el contenido básico de las EEAASS de grado en Arte Dramático y Danza respectivamente.

No obstante, la mayoría de las comunidades autónomas reflejan una estructura precaria para que las enseñanzas artísticas converjan hacia el EEES. Existen notables diferencias entre cada comunidad. Por ejemplo, desde la Administración Andaluza la única respuesta estructural hasta el momento ha sido la Ley 17/2007, de 10 de diciembre, de Educación de Andalucía (LEA) y el Decreto 450/2008, de 9 de septiembre, por el que se regula el Consejo Andaluz de Enseñanzas Artísticas Superiores. La LEA ha establecido los órganos de gobierno de los centros, contemplando la creación además del Consejo Andaluz de Enseñanzas Artísticas Superiores, el Instituto Andaluz de las Enseñanzas Artísticas Superiores (IAEAS). Sin embargo, el IAEAS no ha sido constituido aún, mientras que el funcionamiento del Consejo Andaluz de Enseñanzas Artísticas Superiores es casi inexistente. En contraposición, se encuentra el caso de la Comunitat Valenciana que con la Ley 8/2007, de 2 de marzo, ha sido pionera con la creación del Instituto Superior de Enseñanzas Artísticas (ISEACV), cuyo objeto es la ordenación de los centros públicos que imparten estas enseñanzas en la Comunitat Valenciana. Con ello, la Generalitat Valenciana obtiene un desarrollo legislativo estructural considerablemente más avanzado que el resto de las comunidades autónomas.

Por lo tanto, aunque el espíritu legislativo estatal y autonómico parece que apoya la integración de las EEAASS dentro de la educación superior, lo cierto es que nos encontramos en general con una situación donde la legislación va muy por delante de la situación que viven los centros de enseñanza. Las grandes aspiraciones chocan de frente con una realidad que impide su desarrollo. Una muestra evidente puede verse en las condiciones en las que se desarrolla la investigación en los centros de EEAASS, dentro del ámbito de las disciplinas que les son 
propias. Ya en 1995, la Ley Orgánica sobre Participación, Evaluación y Gobierno de los Centros Docentes (LOPEGC) determinó, por primera vez, la capacidad investigadora de los centros superiores de enseñanzas artísticas. Sin embargo, las condiciones de la mayoría de los centros de enseñanzas artísticas (horarios lectivos del profesorado similar al de profesores de secundaria, inexistencia de despachos departamentales, bibliotecas poco equipadas, etc.) imposibilitan que la investigación pueda ser compatible, por lo que los costes económicos, energéticos y temporales son soportados por los propios profesores. Los centros, en estas condiciones, no facilitan la labor investigadora. Como Jesús Galindo (1998) ha llegado a expresar, "no se puede vivir la experiencia reflexiva en un medio que no promueve las preguntas, o que incluso las inhibe o reprime" (p. 14). A pesar de ello, el número de profesores doctores aumenta cada año.

Por todo lo expuesto anteriormente, es innegable que el marco legal que afecta actualmente a las enseñanzas superiores de danza y arte dramático predispone un futuro prometedor para su integración al EEES. No obstante, la convergencia europea de las EEAASS se enfrenta a un desafío importante, dado el retraso que estas enseñanzas llevan experimentando en su incorporación de hecho al sistema de educación superior. Este desafío afecta no sólo a la estructura de su sistema educativo, tal y como hemos expuesto más arriba, sino que se precisa de un esfuerzo extraordinario para adaptar el currículo a las nuevas exigencias del espacio europeo.

\section{EL DESAFÍO CURRICULAR DE LAS ENSEÑANZAS ARTÍSTICAS SUPERIORES}

El nuevo reconocimiento de las enseñanzas superiores de danza y arte dramático como enseñanzas superiores debe ser considerado como una verdadera oportunidad de mejorar el desarrollo en la danza y el teatro profesional. No obstante, el desarrollo de un currículo para la formación de profesionales que haga frente a las demandas del espacio europeo se ve limitado por su reciente incorporación a la enseñanza superior. Así, el aspecto curricular también se ve afectado por el distinto tratamiento que reciben desde las comunidades autónomas. Mientras, por ejemplo, en Andalucía, Aragón o Asturias, las EEAASS continúan dependiendo de las Consejerías de Educación, desde niveles de gestión comunes a las enseñanzas profesionales artísticas y/o educación secundaria, en otras comunidades (e.g. Comunidad de Madrid, mediante el Decreto 77/2010, del Consejo de Gobierno) las EEAASS se ubican dentro de la Dirección General de Universidades e Investigación, lo que ayudará a que con mayor rapidez se produzca una plena convergencia de las EEAASS con el EEES.

Por otro lado, la definición de un campo de conocimiento no está exenta de sus luchas internas, tal y como ha venido detallando la investigación sobre las disciplinas (Cuesta, 1997; Foucault, 1978; Goodson, 1995). Así, la nueva situación de las EEAASS no ha sido recibida con el mismo entusiasmo por parte de todos los sectores. De hecho, gran parte del profesorado y algunos decanos 
universitarios de enseñanzas artísticas universitarias se mostraban menos partidarios de una integración de las enseñanzas de danza y arte dramático al ámbito universitario. Es al menos como debe entenderse de la protesta hecha pública en la Conferencia de Decanos de Bellas Artes, reunidos el 1 de diciembre de 2009 en Málaga, contra el Real Decreto 1614/2009, donde perciben este nuevo estadio de las EEAASS como una competencia "desleal" hacia determinadas titulaciones universitarias preexistentes al EEES (e.g., licenciatura de bellas artes). De hecho, desde los centros de las EEAASS se podrán emitir grados y posgrados. El mapa de titulaciones parece complicarse aún más si tenemos en cuenta que el mismo apartado 3 del artículo 58 de la LOE ha propiciado que determinadas universidades desarrollen títulos similares o equivalentes a los establecidos en la Ley Orgánica (véase Alejandre, 2010). Esta situación se traduce en conflictos entre diferentes campos de conocimiento, provenientes de la universidad y de las EEAASS. Estos conflictos han derivado en el ámbito judicial, dado que distintas universidades españolas están interponiendo contenciosos administrativos al Real Decreto1614/2009, al entender que los grados emitidos por los centros de EEAASS podrían estar suponiendo una competencia para sus propias titulaciones existentes o futuras.

Lejos de luchas por campos de conocimiento, y salvando las diferencias estructurales, tanto la universidad como los centros de EEAASS deben entender la convergencia europea como un momento para mejorar la educación superior en España. Esta mejora requiere necesariamente la conexión entre la realidad profesional y las necesidades sociales a través de currículos evaluables por agencias de calidad.

Aunque de una manera explícita no se han pronunciado ninguno de los principales documentos de la Unión Europea, la creación de sistemas de acreditación de la calidad homologables entre países es un aspecto fundamental en la creación del EEES (Egido y Haug, 2006). Como bien reconoce Garduño (1999), la calidad requiere un juicio valorativo que viene dado por la evaluación. En la evaluación de la calidad de la enseñanza superior se deben tener en cuenta los propósitos que demande la sociedad, atendiendo a la diversidad y heterogeneidad (Fernández, 2004; Lemaitre, 2004; Tiana, 1996). La evaluación externa es, por tanto, otra novedad de las EEAASS en España. Por ello, sería aconsejable en futuras reflexiones intentar establecer una definición de calidad para estas enseñanzas, atendiendo a sus características específicas y a consensos sobre calidad en educación superior.

El reto que supone la convergencia europea para la educación superior debe ayudar a superar la visión de que la formación de profesionales se limita solo a transmitirles conocimientos y habilidades específicas. Por ello un currículo para las enseñanzas artísticas debe buscar un significado social a corto, medio y largo alcance (Feixas, 2004; Zaldívar, 2005). Se trata de formar futuras personas, para que desde su esfera profesional mantengan la capacidad de desarrollo individual, su capacidad crítica.

Por lo tanto, sobre estas dos finalidades debería basarse el desafío curricular 
al que se enfrentan las enseñanzas artísticas. Por un lado, se trata de capacitar al futuro profesional para dotarle de una competitividad necesaria para el difícil mercado laboral donde ejercerá su función. De otra, consiste en potenciar las capacidades para que esta labor sepa encuadrarla dentro de una convivencia social.

Competencias profesionales y democráticas son necesarias en la formación de las EEAASS. Junto con los conocimientos específicos de danza y arte dramático se pueden desarrollar habilidades de pensamiento social y crítico, que de alguna manera, pueden articularse a través del trabajo en la escena; pero también a través de la crítica, la teoría y la investigación de las artes escénicas. La autonomía del arte, incluido el ejercicio dentro de la danza, la interpretación, la dirección escénica, la creación de textos o el diseño y construcción de elementos escénicos, es necesaria, entendida como la inteligencia aplicada a la dirección de la propia conducta. Como manifiesta Marina (2000) "crear es producir novedades eficaces y descubrir posibilidades en la realidad. Es la poética de la acción que no se limita a expresar artísticamente la realidad, sino que aspira a hacerla realmente bella" (p. 209). Esto indicaría el poder de transformación social que es inherente a las artes escénicas.

\section{PROPUESTAS PARA EL PROCESO DE CONVERGENCIA DE LAS ENSE- ÑANZAS DE ARTE DRAMÁTICO Y DANZA AL EEES}

Hace algunas décadas Philip H. Coombs (1985) reclamaba una necesidad urgente de planificar la educación e invertir en ella de forma decidida por todos los países al unísono para intentar paliar los desequilibrios de oportunidad. Sin duda, el espíritu de Bolonia ha sido éste. En las EEAASS en España la competitividad estará condicionada a la superación de las tensiones expuestas en este artículo. Para que las enseñanzas de arte dramático y danza en nuestro país puedan competir con el resto de Europa se necesita que se comience por reconocerles una atención similar a la que recibe la universidad española. En este reconocimiento deberían intervenir la administración, pero también los centros y su profesorado. No debemos olvidar que el éxito o fracaso de la convergencia de estas enseñanzas al EEES afectará al conjunto del sistema de educación superior español, pues es una parte vinculado a este sistema. En las siguientes líneas sugerimos algunas propuestas para el proceso de convergencia europeo que podrían ser tenidas en cuenta por la administración, los centros y el profesorado.

\section{Propuestas para las administraciones}

A día de hoy existe un retraso en el cumplimiento del Real Decreto 1614/2009 por el que se establece la ordenación de las EEAASS reguladas por la LOE. En el citado decreto se asegura que las EEAASS deberán disponer de autonomía en los ámbitos organizativo, pedagógico y económico, correspondiendo a la Administración dotar de los recursos necesarios para ello, para así garantizar el cumplimiento de los principios que sustentan el EEES. Sin embargo, la autonomía 
es aún precaria, al menos al modo en que funcionan los centros universitarios.

Ante la necesaria autonomía de los centros de EEAASS, tal vez la acción más lógica por parte de la Administración sería la de dotar a éstos de un estatus jurídico-administrativo similar al de la universidad. Esto requeriría por parte del Ministerio de Educación la regulación de las cuestiones referidas a su estructura, organización y funcionamiento. Si esto no ocurre nos tememos que los centros de EEAASS se verán abocados a reproducir la situación mantenida a lo largo de la vigencia de la LOGSE; es decir, seguir adscritos a una normativa de secundaria, dada la ausencia de legislación específica y unificada para las EEAASS.

Por otro lado, la falta de una organización y funcionamiento de los centros de EEAASS, al modo que los centros universitarios, parece estar arrastrando limitaciones en el desarrollo curricular de este tipo de enseñanzas, incluidos la impartición de las enseñanzas de máster y doctorado en condiciones homologadas a la universidad. Sin embargo, la respuesta de las administraciones autonómicas en esta cuestión es desigual y en algún caso inexistente, lo que tiene paralizado el proceso. Por ejemplo, los claustros de los centros de EEAASS en Andalucía han manifestado su descontento por la imposibilidad actual de impartir máster y doctorado. Sin embargo, otras comunidades autónomas se encuentran ya impartiendo másteres y doctorados en convenio con universidades. Por ejemplo, el Máster Oficial Interuniversitario en Estudios Teatrales del Institut del Teatre, en convenio con la Universidad Autónoma de Barcelona y la Universidad Pompeu Fabra.

Por otro lado, mientras algunas comunidades aprueban decretos básicos para la ordenación de estas enseñanzas de cara a su integración en el EEES, el gobierno central aprueba títulos de grado y postgrado de teatro y danza en distintas universidades españolas, lo que, según plantea Vieites (2009), podría poner en peligro la existencia de los centros de EEAASS en las provincias.

En conclusión, entendemos que desde la Administración se deben poner en marcha todos los mecanismos que la legislación ya contempla. El éxito de la convergencia con el EEES dependerá en parte de que las administraciones eviten que las enseñanzas de danza y arte dramático caigan en el olvido y, con ello, en una desigualdad respecto al resto de titulaciones que constituyen la educación superior en España.

\section{Propuestas para los centros de EEAASS}

Los tres pilares de la evaluación de la educación superior derivados de la Declaración de Bolonia son la evaluación del alumnado (o del aprendizaje), la evaluación de la actividad docente y, por último, la evaluación institucional de la docencia. Para fortalecer estos pilares los centros de EEAASS deberán prestar especial atención a dos tareas clave. Por un lado, los centros deberán elaborar e implementar un currículo acorde con el EEES. Por otro lado, los centros no deben descuidar la calidad de la enseñanza que les haga competitivos en el nuevo entorno. 
Para la primera tarea, los centros deberían elaborar currículos con la suficiente información para conocer desde donde se parte y hacia donde quieren dirigirse y por qué. Nos parece importante esta cuestión pues la experiencia de reformas educativas ha mostrado que en ocasiones se han afrontado sin el bagaje suficiente de estudios e investigaciones que diagnostiquen qué es lo que realmente no funciona y lo que merece la pena preservar (Torres Santomé, 2009). De cara a evitar este error, consideramos necesario que los planes de las EEAASS pasen por una fase de experimental, al modo en que ha sido abordado en muchos planes de estudios dentro de la Universidad. Como acertadamente afirma Santos Guerra (2006), una institución cerrada al aprendizaje, hermética con las interrogaciones, asentada en las rutinas y que no aprende de sus errores, simplemente no aprenderá.

En cuanto a la segunda de las tareas, los centros requieren mirar por la calidad de la enseñanza que ofrecen, para lo que necesitan contar con modelos de calidad de programas y centros. El procedimiento para la calidad debe abarcar todas las actividades y funciones de la institución, vinculándola a las necesidades de la sociedad en un ámbito y contexto concreto (Martínez y Riopérez, 2005). Así, la integración de las enseñanzas superiores de danza y arte dramático en España al EEES requiere que los nuevos planes de estudio, el aprendizaje del alumnado, el profesorado y los propios centros sean evaluados por las agencias de calidad. La agencia de evaluación encargada de ello debería ser la ANECA, ya que juega un papel fundamental al dirigir la implantación del programa de evaluación institucional y canalizar la puesta en marcha del programa de convergencia al EEES (Calderón y Escalera, 2008). No obstante, adoptar un sistema de evaluación que cuide la calidad de la enseñanza implica no sólo cambios institucionales, sino también un cambio en los valores, creencias y pensamiento de sus agentes.

\section{Propuestas para profesores e investigadores}

Como ya apuntábamos en apartados anteriores, parece prioritaria una equiparación laboral del profesorado de EEAASS con el resto de docentes del ámbito universitario. Esto implicaría asumir que la función del profesorado ya no puede ser sólo docente, sino que debe asumir un cambio de mentalidad para integrar las funciones propias de la investigación. No se debe obviar que la enseñanza superior es el resultado del cruce de la investigación, la educación y la innovación, y que la labor del profesor se encuentra entre la orientación a los alumnos, la investigación y la docencia. Como indica Calderón y Escalera (2008) sin alguna de estas funciones la profesión de docente en educación superior no sería completa.

Integrar las EEAASS en la educación superior implica un cambio profundo en la tarea de su profesorado. Mientras las universidades, desde su creación en el siglo XII, se han dedicado a preservar, crear y transmitir conocimientos y cultura (Mayorga, 1999), los conservatorios y escuelas artísticas en España arrastran una historia más reciente y más alejada de la teorización de su actividad profesional. Así, la totalidad del profesorado debe familiarizarse con los paradigmas, 
metodologías y métodos de investigación consolidados en las universidades y escasamente utilizados en al ámbito artístico hasta la fecha. No obstante, esto no implica renunciar en absoluto a su especificidad en el contenido y en su historia. Como sabemos, las enseñanzas artísticas han focalizado tradicionalmente su actividad investigadora desde la creación artística y performativa. La investigación creativa-performativa se basa en un proceso creativo y/o interpretativo desarrollado por parte del propio artista, de tal modo que es su experiencia artística la que es objeto de estudio (Hernández, 2008). La idea sería encontrar una integración entre la teorización y la praxis, pues estamos de acuerdo con Zaldívar (2005, 2006), en que el carácter creativo y performativo de la investigación artística debiera ser reconocido, equiparable, acreditable y evaluable, tal como es tratada otro tipo de investigación dentro de la universidad.

Queda camino por recorrer para la convergencia europea de los estudios superiores. Es nuestro deseo que la posibilidad de reforma que la educación superior en Europa brinda a las escuelas superiores de danza y arte dramático no se emprenda únicamente para cambiar las etiquetas y otras formalidades de estos estudios. En palabras de Teichler (2006, 71), esperamos que el espacio europeo de educación superior sea para las enseñanzas artísticas "algo más que una frase y algo menos que un sistema". Este artículo ha brindado algunas propuestas a partir de la situación en la que estas enseñanzas se encuentran hoy día. No obstante, la materialización de estas propuestas supone una implicación conjunta de administración, centros y profesorado. Esperemos que podamos ver pronto una armoniosa conjunción en los diferentes niveles de acción.

\section{REFERENCIAS}

Alejandre, M. B. (2010). El abandono de las enseñanzas artísticas superiores. Revista Ampe Andalucía, 164, 28-30.

Beltrán Llavador, J., Hernández i Dobon, F. J. y Montané López, A. (2008). Tradición y modernidad en las políticas educativas en España: Una revisión de las últimas décadas. Revista Iberoamericana de Educación, 48, 53-71.

Calderón Patier, C. y Escalera Izquierdo, G. (2008). La evaluación de la docencia ante el reto del Espacio Europeo de Educación Superior. Educación XX1, 11, 237-256.

Coombs, P. H. (1985). La crisis mundial en la educación. Perspectivas actuales. Madrid: Santillana.

Cuesta, R. (1997). Sociogénesis de una disciplina escolar: la Historia. Barcelona: Ediciones Pomares-Corredor

Decreto 450/2008 por el que se regula el Consejo Andaluz de Enseñanzas Artísticas Superiores (2008, 9 de Septiembre). Boletín Oficial de la Junta de Andalucía (BOJA), 189, Septiembre 22, 2008.

Decreto 77/2010 del Consejo de Gobierno, por el que se modifica la estructura orgánica de la Consejería de Educación (2010, 28 de Octubre). Boletín Oficial de la Comunidad de Madrid (BOCM), 263, Noviembre 3, 2010. 
Egido Gálvez, I, y Haug, G. (2006). La acreditación como mecanismo de garantía de calidad: Tendencias en el Espacio Europeo de Educación Superior. Revista Española de Educación Comparada,12, 81-112.

Feixas, M. (2004). De Bolonia a Berlín. Revista Interuniversitaria de formación del profesorado, 18, 149-162.

Fernández Lamarra, N. (2004). Hacia la convergencia de los sistemas de educación superior en América Latina. Revista Iberoamericana de Educación, 35, 39-71.

Font, J. (2010). Crónicas de un trayecto. En VV.AA., Las Enseñanzas Artísticas Superiores Legislación (pp. 11-16). Murcia: Asociación Española de Centros Superiores de Enseñanzas Artísticas (ACESEA).

Foucault, M. (1978). Las palabras y las cosas. Una arqueología de las ciencias humanas. Madrid: Siglo XXI.

Galindo Cáceres, J. (1998). La lucha de la luz y la sombra. En Galindo Cáceres, J. (Coord.), Técnicas de investigación en sociedad, cultura y comunicación (pp. 9-31). México: Pearson Educación.

Garduño Estrada, L. (1999). Hacia un modelo de evaluación de la calidad de instituciones de educación superior. Revista Iberoamericana de Educación, 21, 93-103.

Goodson, I. F. (1995). Historia del currículum. La construcción social de las disciplinas escolares. Barcelona: Pomares-Corredor.

Hernández Hernández, F. (2008). La investigación basada en las artes. Propuestas para repensar la investigación en educación. Educatio Siglo XXI, 26, 85118.

Lemaitre, M. J. (2004). Redes de agencias de aseguramiento de la calidad de la educación superior a nivel internacional y regional. Revista Iberoamericana de Educación, 35, 73-87.

Ley 17/2007 de Educación de Andalucía (2007, 10 de Diciembre). Boletín Oficial de la Junta de Andalucía (BOJA), 252, Diciembre 26, 2007.

Ley 8/2007 de Ordenación de Centros Superiores de Enseñanzas Artísticas y de la creación del Instituto Superior de Enseñanzas Artísticas de la Comunitat Valenciana (2007, 2 de Marzo). Diario Oficial de la Comunitat Valenciana (DOCV), 5466, Marzo 8, 2007.

Ley Orgánica 1/1990 de Ordenación General del Sistema Educativo (LOGSE) (1990, 3 de Octubre). Boletín Oficial del Estado (BOE), 238, Octubre 4, 1990.

Ley Orgánica 10/2002 de Calidad de la Educación (LOCE) (2002, 23 de Diciembre). Boletín Oficial del Estado (BOE), 307, Diciembre 24, 2002.

Ley orgánica 2/2006 de Educación (LOE) (2006, 3 de Mayo). Boletín Oficial del Estado (BOE), 106, Mayo 4, 2006.

Ley Orgánica 9/1995 de la Participación, la Evaluación y el Gobierno de los Centros Docentes (LOPEGC) (1995, 20 de Noviembre). Boletín Oficial del Estado (BOE), 278, Noviembre 21, 1995.

Los Decanos de Bellas Artes denuncian que el Ministerio de Educación legisla en contra de los estudios universitarios (2009, 2 de Diciembre).Actualidad 
Universitaria. Extraído el 15 de enero de 2010 desdehttp://www.actualidaduniversitaria.com/2009/12/los-decanos-de-bellas-artes-denuncian-que-elministerio-de-educacion-legisla-en-contra-de-los-estudios-universitarios/.

Marchesi, A. (2001). Presente y futuro de la reforma educativa en España. Revista Iberoamericana de Educación, 27, 57-76.

Marina, J.A. (2000). Crónicas de la ultramodernidad. Barcelona: Anagrama.

Martínez Mediano, C., y Riopérez Losada, N. (2005). El modelo de excelencia en la EFQM y su aplicación para la mejora de la calidad de los centros educativos. Educación XX1, 8, 35-65.

Mayorga, R. (1999). Los desafíos de la universidad latinoamericana en el siglo XXI. Revista Iberoamericana de Educación, 21, 25-40.

Pastora, J. F. (2000). La LOGSE y la LOPEGCE. Barcelona: CISS Praxis Educación.

Pliego de Andrés, V. (2008). Presente y futuro de las enseñanzas artísticas superiores. Periódico Escuela, 3845 (1607), 7.

Real Decreto 1614/2009 por el que se establece la ordenación de las enseñanzas artísticas superiores reguladas por la Ley Orgánica 2/2006, de 3 de mayo, de Educación (2009, 26 de Octubre). Boletín Oficial del Estado (BOE), 259, Octubre 27, 2009.

Real Decreto 303/2010 por el que se establecen los requisitos mínimos de los centros que impartan enseñanzas artísticas reguladas en la ley Orgánica 2/2006, de 3 de mayo, de Educación (2010, 15 de Marzo). Boletín Oficial del Estado (BOE), 86, Abril 9, 2010.

Real Decreto 365/2007 por el que se regula el Consejo Superior de Enseñanzas Artísticas (2007, 16 de Marzo). Boletín Oficial del Estado (BOE), 81, Abril 4, 2007.

Real Decreto 630/2010 por el que se regula el contenido básico de las enseñanzas artísticas superiores de Grado en Arte Dramático establecidas en la Ley Orgánica 2/2006, de 3 de mayo, de Educación (2010, 14 de Mayo). Boletín Oficial del Estado (BOE), 137, Junio 5, 2010.

Real Decreto 632/2010 por el que se regula el contenido básico de las enseñanzas artísticas superiores de Grado en Danza establecidas en la Ley Orgánica 2/2006, de 3 de mayo, de Educación (2010, 14 de Mayo). Boletín Oficial del Estado (BOE), 137, Junio 5, 2010.

Santos Guerra, M. A. (2006). La Escuela que Aprende. Madrid: Ediciones Morata.

Teichler, U. (2006). El Espacio Europeo de Educación Superior: Visiones y realidades de un proceso deseable de convergencia. Revista Española de Educación Comparada, 12, 37-79.

The Bologna Declaration (1999, 19 de Junio). Joint declaration of the European Ministers of Education. The European Higher Education Area, 1999.

Tiana, A. (1996). La evaluación de los sistemas educativos. Revista Iberoamericana de Educación, 10, 37-61.

Torres Santomé, J. (2009). Obviando el debate sobre la cultura en el sistema 
educativo: Cómo ser competentes sin conocimientos. En Gimeno Sacristan, J. (Comp.), Educar en competencias: ¿qué hay de nuevo? (pp. 143-175). Madrid: Morata.

Vélaz de Medrano Ureta, C. (2008). Formación y profesionalización de los orientadores desde el enfoque de las competencias. Educación XX1, 11, 155-181.

Vieites, M. F. (2009). Varapalo a las enseñanzas teatrales. ADE Teatro, 127, 7-8.

Zaldívar, A. (2005). Las enseñanzas musicales y el nuevo marco europeo de educación superior. El reto de un marco organizativo adecuado y la necesidad de investigación creativa y "performativa". Revista Interuniversitaria de formación del profesorado, 19, 95-122.

Zaldívar, A. (2006). El reto de la Investigación creativa y "performativa". Eufonía: Didáctica de la Música, 38, 87-94. 